\title{
Prácticas educativas familiares en el desempeño escolar ${ }^{1}$
}

\author{
Liliana Belalcazar Pérez ${ }^{2}$ \\ Ángela Delgado Caicedo ${ }^{3}$ \\ Consideración ${ }^{4}$
}

\begin{abstract}
Resumen
La presente investigación buscó correlación entre prácticas educativas familiares y desempeño escolar. Para este trabajo, se tomó como muestra, 208 estudiantes de los grados cuartos de básica primaria, de la Institución Educativa Municipal (I.E.M.) María Goretti. Es un estudio de corte cuantitativo; se empleó como instrumento, una escala elaborada y validada para tal fin. La información se analizó con el programa estadístico para ciencias sociales SPSS. En general, las prácticas educativas familiares son variadas. Se encontraron que las prácticas educativas son diversas, sin embargo, se pudo establecer que las prácticas educativas familiares no se correlacionan con el desempeño escolar.
\end{abstract}

Palabras clave: familia, desempeño, estudiantes, prácticas, lenguaje, hábitos.

\section{Educational and familiar practices in the school performance}

\begin{abstract}
This research seeks a correlation between family educational practices and school performance. For this work, a sample was taken, 208 fourth grade students from the primary school Institución Educativa Municipal (I.E.M.) María Goretti. It is a quantitative study; a developed and validated scale was used as an instrument for this purpose. Data were analyzed with the Statistical Package for Social Sciences SPSS. In general, family educational practices are varied. They thought that the educational practices are diverse, nevertheless, it was possible to establish that the educational familiar practices do not correlate with the school performance.

Keywords: family, performance, students, practice, language, habits.
\end{abstract}

1 Recibido: 06 de noviembre de 2012. Aceptado: 16 de enero de 2013

2 Liliana Belalcazar Pérez. Magister en educación desde la diversidad. Licenciada en Comercio y Contaduría, egresada de la Universidad Mariana; Docente de básica primaria, Institución Educativa Municipal La Rosa. Pasto- Nariño. Correo electrónico: lilibelper@hotmail.com

3 Ángela Delgado Caicedo. Bióloga. Magister en educación desde la diversidad. con énfasis en Microbiología, egresada de la Universidad de Nariño; Docente de básica primaria, Institución Educativa Municipal María Goretti. Pasto-Nariño. Correo electrónico: angeladelgado97@yahoo.es

Tutor orientadora: Dra. Dolly Vargas García 


\section{Justificación}

De los agentes de socialización, la familia es muy importante en el desarrollo, por tanto, también es evidente la importancia de su participación en la generación de actividades que apoyen el aprendizaje y en la inclusión de prácticas de lenguaje, así como su papel en la formación de hábitos, dado que las relaciones entre padres e hijos condicionan sus reacciones inmediatas y esto incluye el entorno escolar, de tal modo que si no se cuentan con prácticas educativas familiares positivas, puede verse afectado el desempeño escolar.

En esta investigación, el desempeño escolar se considera un indicador de lo aprendido por una persona para su formación integral, convirtiéndose en una tarea tanto del docente como de la familia, quien es la primera en ayudar a formar hábitos en sus hijos, como parte de sus prácticas educativas familiares: actividades de aprendizaje, prácticas de lenguaje y hábitos en el hogar, que favorecen el desempeño escolar. Así lo han demostrado estudios realizados Peralbo, Fernández (2003), Gonzáles, Núñez, Álvarez, Roces, y Valle (2003), Alguacil, Pañellas (2009), entre otros, quienes afirman que existe relación entre la participación familiar en el campo educativo y el éxito académico de los estudiantes.

Por tal razón, es imprescindible promover en los padres de familia, prácticas educativas familiares, fruto del contexto, coherentes con la época. Sin embargo, es importante considerar que existen familias con deseo de ayudar en los quehaceres escolares de sus hijos, pero, a veces carecen de condiciones necesarias para hacerlo.

Esta investigación, pretende conocer la relación entre prácticas educativas familiares y desempeño escolar, para beneficiar a la comunidad educativa en general, porque al determinar si existe relación entre las prácticas educativas familiares y el desempeño escolar, se pueden derivar estrategias para tener en cuenta desde la infancia y poder garantizar un mejor desempeño escolar en los estudiantes.

\section{Antecedentes}

En el desempeño escolar, ha cobrado importancia el papel de los padres de familia; esto ha llevado a que las investigaciones relacionadas con las prácticas educativas familiares, hayan aumentado y generen interés en aquellas personas y entidades relacionadas con la educación.

En el presente artículo se citan varias investigaciones relacionadas con prácticas educativas familiares y desempeño académico, el impacto que tienen los diferentes tipos de conductas y patrones de comportamiento de los padres, implicación de las familias en los institutos de enseñanza, la influencia de la familia en la educación de los menores, sociedad, familia y aprendizaje, el papel de los contextos hogareños y prácticas alfabetizadoras familiares, que enriquecen teóricamente este estudio investigativo.

Peralbo, Fernández (2003), analiza en un estudio, el contexto familiar de aquellos alumnos y alumnas que presentan un bajo rendimiento escolar. Los datos fueron obtenidos de una muestra de 627 estudiantes de $2^{\circ}$ y 665 de $4^{\circ}$. Al cruzar la variable preocupación o contacto de los padres con el centro escolar, con la asistencia a clases particulares, encuentran que el hecho de que el centro escolar llame a las familias no resulta significativo a la hora de poner en marcha posibles recursos compensadores como puede ser la asistencia a clases particulares.

Manifiestan que sólo pueden establecer relaciones significativas cuando las familias acuden por iniciativa propia al centro educativo para interesarse por el rendimiento académico de sus hijos/as y al poner en marcha mecanismos diversos para intentar paliar las dificultades que a los estudiantes se les presente.

Gonzales, Núñez, Álvarez, Roces, y Valle (2003), desarrollaron un estudio 
para conocer el impacto que tienen los diferentes tipos de conductas y patrones de comportamiento de los padres en el rendimiento académico de sus hijos. Confirmaron que la percepción por parte de los hijos de comportamientos autorregulados de sus padres influye significativamente sobre su rendimiento académico.

Alguacil, Pañellas (2009), dan a conocer una investigación en la que analizan la implicación de las familias de estudiantes de 12 a 16 años en los institutos de enseñanza secundaria, mediante su participación en las Asociaciones de Madres y Padres de Alumnos y en las actividades organizadas por el propio instituto.

Las conclusiones, confirman que la participación de los padres y madres en la escuela produce consecuencias beneficiosas para toda la comunidad educativa: actitudes colaborativas de los padres y de las madres; valoración positiva del docente; mayor autoestima, mejor rendimiento de los alumnos y mayor compromiso con el entorno.

Espitia y Montes (2009) realizaron un estudio para analizar la influencia de la familia en la educación de los menores del barrio Costa Azul de Sincelejo (Colombia). Tuvieron en cuenta las características socioeconómicas, culturales y educativas del entorno familiar y la identificación de prácticas, visiones, significados y expectativas con respecto a la educación de sus hijos.

Los resultados revelaron que los padres atribuyen importancia a la educación desde sus visiones, expectativas y significados, pero carecen de condiciones necesarias para impulsar procesos; sus prácticas educativas, recursos, hábitos, tiempo, responsabilidades son limitados, lo cual es un obstáculo para el éxito en el aprendizaje de los hijos.

Querejeta (2010) investigó sobre las relaciones entre sociedad, familia y aprendizaje. Particularmente se refiere a las características del contexto alfabetizador hogareño de poblaciones carentes y sus repercusiones en las habilidades y conocimientos pre lectores de sus hijos.

Resumiendo, los hallazgos de esta investigación dan cuenta, por un lado, de la variabilidad presente en los contextos hogareños de la pobreza, en los conocimientos y habilidades pre lectores infantiles. Los padres y madres informaron sobre la presencia en un porcentaje relativamente alto de características y prácticas adecuadas, que pueden incidir favorablemente sobre el aprendizaje del lenguaje escrito.

Hincapié (2011), realizó una investigación descriptiva, con el objetivo de diferenciar las prácticas alfabetizadoras familiares de acuerdo con los tipos de familia (mono parental, extensa y nuclear). La investigadora encontró: que las prácticas alfabetizadoras familiares son menos favorables en las familias pertenecientes a estratos bajos, las prácticas alfabetizadoras en general fueron más utilizadas por las familias nucleares. Las prácticas de lecturas de cuentos, uso de portadores de texto, son escasas en las familias independientemente al estrato y tipo de familia.

Las prácticas de lenguaje son las más usadas por las familias en comparación con las demás prácticas; en la familia nuclear se evidenció más uso de prácticas alfabetizadoras familiares, como el uso de lenguaje que en la familia mono parental y extensa. Las familias desconocen la importancia de algunas prácticas que están fuertemente relacionadas con los procesos lectoescriturales, tales como ir a la biblioteca, leer con sus hijos, tener material de lectura en casa, compartir momentos de lectura con los hijos entre otros.

Las investigaciones de Querejeta (2010) sobre las relaciones entre sociedad, familia y aprendizaje, al igual que la de Hincapié (2011), tienen como objetivo diferenciar las prácticas alfabetizadoras familiares de acuerdo con los tipos de familia (mono parental, extensa y nuclear), aportan a la presente investigación, el 
instrumento que relacionan la familia y desempeño escolar; objeto de estudio de este trabajo de investigación, se observan las relaciones del desempeño escolar con: nivel educativo de los padres, apoyo en las tareas, participación de los padres en la escuela, importancia de los padres en la educación, variabilidad económica en los contextos hogareños, tipos de familias y prácticas lectoras, las cuales están relacionadas con las prácticas educativas familiares.

\section{Problema de investigación}

¿Cuáles son las relaciones entre prácticas educativas familiares y desempeño escolar en básica primaria?

\section{Objetivo general}

Establecer relaciones entre prácticas educativas familiares y desempeño escolar en básica primaria

\section{Objetivos específicos}

- Identificar las prácticas educativas familiares en estudiantes de los grados cuartos de básica primaria de la I.E.M. María Goretti.

- Describir el desempeño escolar de las estudiantes en estudio.

- Comparar el desempeño escolar con las prácticas educativas familiares para establecer sus relaciones.

\section{Descripción teórica}

Tanto a la familia como a la escuela, les interesa mejorar el desempeño escolar de los estudiantes. Las prácticas educativas familiares están influenciadas por la cultura y el nivel económico de los padres y las madres.

\section{Prácticas educativas familiares}

\author{
Para Alfonso y Amat (2003, 32) \\ Las prácticas educativas familia- \\ res no constituyen un todo homogé- \\ neo. Por el contrario, se caracterizan
}

por su heterogeneidad, es decir, no hay dos familias que hagan exactamente las mismas cosas, sino que una misma familia modifica sus comportamientos a lo largo del tiempo, de modo que sus hijos se sumergen en experiencias diferentes.

Al igual que la diversidad de prácticas educativas familiares, cuando se trata de relaciones de la familia con los hijos, existen diferentes terminologías que pueden ocasionar confusiones, por tal razón, se procede a presentar los conceptos con los que se trabaja en la presente investigación:

Las prácticas hacen referencia a las acciones y comportamientos concretos que se privilegian y se construyen en las relaciones interhumanas de la vida cotidiana. El trato, por su parte, hace referencia a la calidad y estilo del cuidado; las pautas responden a las orientaciones que los padres/madres priorizan como ideal y guía en las relación de formación. Los patrones de crianza hacen referencia a las costumbres, la tradición y el acervo cultural que se transmiten entre una generación y otra. (Botero, Salazar y Torres, 2009, 30)

Se puede afirmar entonces que las prácticas surgen dentro de la familia como consecuencia de las relaciones sociales, de la cultura que se ve reflejado en sus costumbres. Completa el concepto Henao Ramírez (2007)

Se entiende por prácticas educativas familiares aquellas preferencias globales de comportamiento de los padres o figuras de autoridad relacionadas con las estrategias educativas encausadas hacia los hijos, las cuales poseen como característica, la bidireccionalidad en las relaciones padres-hijos, debido a que los actos de los padres generan consecuencias sobre los hijos así como las acciones de los hijos influyen sobre los padres. (235). 
En ese mismo sentido, define la educación familiar como: "la acción de criar y educar a un niño o niños, realizada por adultos en el seno de grupos familiares, padres de los niños implicados" (785), es decir, que dentro de la familia inicia el proceso de educación el cual va a ser complementado posteriormente por el trabajo que se desarrolla en las instituciones educativas.

La importancia de la familia ha sido tema de numerosos campos y autores, Redding (sf: 5), por ejemplo afirma:

El ambiente familiar, denominado el currículum del hogar, es un compendio de las conversaciones deliberadas que mantienen padres e hijos sobre el colegio y sobre los acontecimientos de la vida diaria como: estimular la lectura en el tiempo libre y comentar lo leído; guiar y revisar desde una postura crítica lo que se ve en la televisión y las actividades con los amigos y compañeros; aplazar las gratificaciones inmediatas para poder llegar a alcanzar metas a largo plazo; expresar afecto e interés por los aspectos académicos de los hijos y por otros progresos en su desarrollo personal; y quizá, entre los esfuerzos continuos que se pueden realizar.

Este autor hace énfasis en la comunicación como estímulo a la lectura y agrega: leerles a los niños y comentar con ellos aspectos de la vida diaria de los centros educativos, es decir, constituye una práctica que no solo mejora las relaciones familiares sino que crea motivación hacia el lenguaje oral y escrito y en consecuencia, al entorno escolar.

La lectura está asociada a la escuela y a la familia, los niños pueden aprender a sentir gusto por ella, cuando desde el hogar ha sido valorada y dedica cierto tiempo a leer, esos comportamientos y actitudes de los miembros de la familia, permiten que se empiecen a generar hábitos lectores en los niños. El hecho de que un niño vea leer a su padre, genera en él hábitos de lectura. Por tanto es necesario fortalecer el papel de la familia en el desempeño escolar de los estudiantes.

Derivado de las consideraciones anteriores, es válido reafirmar que la educación de un individuo no comienza cuando este ingresa a un centro escolar. Se inicia desde la familia y es ahí donde se deben proporcionar, al igual que en la escuela, estrategias y prácticas educativas que contribuyan a la formación del individuo. Para Alfonso y Amat (2003, 3): "Las instituciones escolares no han cambiado al ritmo con que lo ha hecho la institución familiar en estas últimas décadas. La escuela se ve obligada a integrar nuevas formas de organización familiar, tan variadas y diversas como la sociedad que nos rodea"

Del planteamiento anterior se deduce, que la escuela debe incorporar métodos que permitan estar acorde con los cambios sociales, familiares e individuales, de tal manera que se dé un diálogo permanente y claro entre la escuela y la familia con un objetivo en común: el desarrollo del individuo. La familia tradicional y nuclear ha cambiado radicalmente su forma y manifestaciones, ya que hay hogares donde la cabeza de hogar es únicamente la madre, o los hijos están a cargo de los abuelos, en algunos casos son responsabilidad de los hermanos mayores. En fin hay tantas formas de familias que requieren de una comprensión clara por parte de los centros escolares.

En la tradición de las distintas comunidades se mantienen prácticas educativas familiares relativas a la crianza de los niños y las niñas que han sido transmitidas de las personas más mayores a las más jóvenes. Sin embargo, las actuales familias nucleares- especialmente en las grandes ciudades- tienen muchos menos apoyos sociales para hacer efectiva su labor de padres. (Vila, 1998, 163) 
Sobre la base de las consideraciones anteriores, una vez más, la cultura forma parte importante en las prácticas educativas familiares.

A la luz de la teoría, se puede entender que las practicas familiares contribuyen a fortalecer características personales de los hijos, así como también: "Ayudar a resaltar las prácticas educativas valiosas que se dan en la vida diaria, para potenciar esas situaciones y fomentar los sentimientos de competencia, autonomía y autoestima" (Paz, 2000, 78-79) necesarias para un buen desempeño escolar.

Es importante tener en cuenta que la familia debe tomar el control del proceso de formación de sus hijos, Marín (2004, 58) "El rol del padre de familia debe estructurarle para la vida diaria a su hijo, esto implica, vigilar sus hábitos de estudio, inculcar seguridad en sí mismo y enseñarlo a valorarse, aceptarse y quererse". De manera que aprendan a interactuar en los diferentes contextos y se beneficien con las situaciones que se les presente, en ningún momento desaprovechen sus propias potencialidades.

Las diferentes prácticas deben ser adaptadas a las capacidades y al contexto donde se desarrolla el niño, para lograr un desempeño de manera eficiente en el diario vivir. "El ajuste de nuestra prácticas a nuestra capacidad de desempeño y a nuestro medio ambiente determina el grado de eficacia que tendremos en nuestras rutinas cotidianas" (Gary, 2004, 78). La familia cuando desarrolla prácticas educativas, debe tener en cuenta las características individuales de sus hijos lo que significa que estas prácticas deben estar basadas en sus habilidades, aptitudes y limitaciones.

Casi siempre, las prácticas educativas son transmitidas a los hijos, por eso es importante, a través de ellas, enseñarles a valorar la educación, estar presente en sus triunfos y sus fracasos, incluirlos con mayor fuerza en el proceso de aprendizaje. Ortega (2006, 306). "uno no nace con predisposiciones contra el estudio, sino que en el ámbito familiar "micro cultural" se modula qué predisposiciones se cultivan o se refuerzan, qué red de expectativas para el individuo se entreteje". En el ámbito escolar se cree que debe haber una buena predisposición para aumentar los conocimientos, los cuales van a estar relacionados con el desempeño escolar y este a la vez con las prácticas educativas familiares como algo importante en el proceso de aprendizaje.

En las prácticas educativas familiares, además de prácticas lectoras, también se debe tener en cuenta el contacto entre padres e hijos y su relación afectiva, de modo que puedan establecer unos hábitos que respeten su individualidad y favorezcan el aprendizaje; los progenitores deben conocer constantemente las acciones de sus hijos dentro de las instituciones o centros educativos donde estos se están formando.

Los estudios han relacionado positivamente ciertas prácticas familiares con el aprendizaje del niño, algunas se refieren a la relación padre-hijo (expresiones de afecto, conversar diariamente, hacer comentarios en familia sobre libros, revistas, visitas a museos); otras se refieren a las rutinas familiares (marcar un tiempo de estudio en casa; disponer de un lugar para leer $y$ estudiar...); $y$ otras, a las expectativas familiares y el control (dar prioridad al trabajo escolar y a la lectura sobre ver televisión; controlar el grupo de amigos de los hijos, conocer el progreso de sus hijos en la escuela). (Carrasco, 2007, 178).

En consecuencia, las familias son un punto clave para la formación integral del niño, su participación debe ser constante, sin limitarse únicamente a garantizar condiciones económicas las cuales son necesarias pero no suficientes. Se requiere que los progenitores estén en un contacto continuo con sus hijos. 


\section{Prácticas educativas familiares del lenguaje}

Los seres humanos tienen diferentes formas de comunicación, entre ellas la oralidad, como primera forma para comunicarse, da origen a la escritura, la cual es muy importante para que el ser humano consigne su historia, le permite guardar de manera permanente toda la información que desee. El lenguaje, acompaña a las personas durante toda su vida; les permite mantenerse informadas e intelectualmente activas.

El lenguaje escrito forma parte de la realidad en la que el niño se desarrolla, hay lenguaje escrito en la calle, en la ropa, en las bolsas del supermercado, en carteles, en los autobuses, en las tiendas..., pero es necesario que vea a los adultos utilizar activamente el lenguaje escrito para interesarse por él. (Cerillo, Larrañaga y Yubero, 2002, 13)

Después de las consideraciones anteriores, el rol de la familia en el proceso de aprendizaje del lenguaje escrito, se puede tomar como el mecanismo que relaciona a los estímulos externos como: letreros, revistas, mensajes que están en la calle, y el proceso de aprendizaje de lenguaje escrito del niño, si este se usa por la familia, el niño comienza a hacerlo también, Además, "Las prácticas de lectura y escritura de los padres también parecen estar relacionadas con el rendimiento académico de los hijos." (Gómez, 2001, 93). Prácticas que se realizan no necesariamente desde los libros, sino desde el contexto donde el hijo realiza todas sus actividades habituales, si el niño ve en a sus progenitores a unos sujetos que analizan e interpretan todo aquello que está escrito, están constantemente motivados por la lectura y la escritura, además les gusta visitar las bibliotecas, es posible que él también se sienta atraído y le agrade esta forma de lenguaje.
Se trata de estimular y mantener la motivación por el aprendizaje y el desarrollo del lenguaje escrito, en sus dos vertientes producción y recepción (escribir y leer): esta afirmación está referida tanto al niño aprendiz como al entorno del niño, ya que "los otros" son los agentes sociales que interactúan con él y le sirven de modelo. Y en la primera infancia los padres son los auténticos héroes de sus hijos, todo lo que hacen y lo que dicen lo quieren imitar los niños. (16)

Es importante que en casa aprendan a dialogar sobre los acontecimientos diarios, permitirle al niño que exprese sus inquietudes, realizar actividades de juego; de alguna manera mantenerlos motivados para que sientan emoción al participar en actividades de diálogo o de lenguaje escrito.

El sentimiento de las primeras lecturas conjuntas, el placer que provoquen en los niños, la emoción que produzcan, el bienestar que experimenten en las distintas situaciones de lectura, el tono afectivo que rodee la situación de leer, marcarán la motivación de los niños hacia los libros y la lectura. También se verá influida porel contexto más amplio, por un ambiente que les invite o les aleje de los libros. En un principio será una motivación externa: atención de los adultos, situación de juego, refuerzo afectivo de los padres y/o del profesor... pero necesariamente debe transformarse en una motivación interna, en el placer de leer por leer. (16)

De manera que en edad escolar el niño no únicamente debe dedicarse a leer aquello que le dejan del colegio, debe conservar el hábito de la lectura, deleitándose de esta y compartiendo con las personas más allegadas a él.

El orden familiar de identificaciones nos ha revelado que una identificación con una persona que mantiene una relación satisfactoria 
y placentera con unos valores más o menos académicos: gusto por la lectura, la poesía, alguien que guarda con celo sus cartillas de escolaridad, que habla de su escolaridad como la mejor etapa de su vida, una autodidacta que consulta sus libros...etc., hace de puente y puede traspasar estos gustos al escolar. Observamos una relación positiva entre gusto por la lectura y rendimiento académico (este gusto suele coincidir en madre e hija, la hija se identifica con la madre $y$, a través de ella, con la lectura) (Gómez, 2001, 93)

Es necesario tener en cuenta que los hábitos son disposiciones que se adquieren desde los hogares, para la realización de determinados actos, es decir son las repeticiones de diferentes actividades que conllevan a resultados positivos, en el caso de estudiantes puede ser a un desempeño escolar alto.

\section{Hábitos en el hogar}

El hábito es un modo de conducta adquirido, es decir, una reacción aprendida, que supone la tendencia a repetir y a reproducir ciertas acciones o actuar en la misma forma general bajo las mismas o similares circunstancias. La utilidad real del hábito radica en el hecho de que proporciona una razonable variabilidad de respuestas a situaciones que son básicamente semejantes. Es la tendencia común en todos los seres humanos, a adquirir modos fijos de reacción ante situaciones determinadas. (Kelly, 1982, 171).

Existe cierta relación entre el contexto familiar y el desempeño escolar, muchos hábitos y las actitudes que asumen las familias inciden en el desarrollo que puedan alcanzar sus hijos en las instituciones educativas. Hoy en día se ve la necesidad que las y los estudiantes presenten una mejor disposición hacia la lectura, tratando de que logren alcanzar una comprensión en esta, facilitándoles el entendimiento en diversas áreas.

Los resultados confirman mayores niveles de competencia en el alumnado cuyos padres presentan mejores actitudes hacia la lectura y declaran dedicar mayor número de horas semanales a esta actividad. Se concluye afirmando el interés de estas variables para caracterizar el contexto socio familiar en que se desenvuelve el alumno y valorar, en función de éste, los resultados de aprendizaje logrados en las instituciones escolares.(Gil, 2009, 301)

El compromiso de los padres de familia es fundamental para las instituciones educativas, debe presentarse una colaboración entre padres y profesores, porque ambos son decisivos en la educación de los niños.

Dentro de un aula de clase se presentan muchas diferencias en el rendimiento de los estudiantes, algunos están relacionados con experiencias de aprendizaje, actitudes y expectativas que están presentes en las familias.

Son numerosas las características que podrían ser consideradas en la unidad familiar a la hora de analizar su contribución al aprendizaje escolar de los hijos. Además de la colaboración e implicación de los padres en la escuela, orientada al logro de unos objetivos educativos y de aprendizaje comunes, se han considerado las características estructurales y de funcionamiento de la familia, y el ambiente educativo del hogar como factores que influyen en los resultados escolares. (303)

En el desarrollo de las prácticas educativas, la importancia que muestran los padres hacia la escuela y sus hijos, son necesarios para mejorar el desempeño escolar.

Existen prácticas que pueden ayudar a los estudiantes a desenvolverse mucho mejor en el contexto escolar, "Los hábitos y actitudes observadas en los padres influyen en la adquisición de hábitos y el 
desarrollo de actitudes por los hijos en edades tempranas" (316). Lo común en las prácticas educativas familiares está dado por el grado de control de los padres en la relación con sus hijos, la comunicación entre los integrantes del hogar, el conocimiento del contexto que rodea a los hijos y el afecto, son aspectos que se reflejan en el contexto escolar.

\section{Desempeño escolar}

Con referencia al desempeño escolar y rendimiento académico se tienen en cuenta algunos autores como: Gonzáles (2005), Rodríguez y Tejedor (1996) y Paz (2007), quienes aportan varias interpretaciones. Algunas de ellas son:

El desempeño escolar se define como el dominio por parte de los alumnos de los objetivos correspondientes al subsistema educativo al que pertenece. Se consideran sus indicadores diferentes estados por los que puede transitar el alumno como la condición de regular/irregular, número de materias aprobadas/ reprobadas, número de exámenes presentados para acreditar una asignatura, número de créditos acumulados y calificaciones obtenidas. Gonzáles (2005, 33)

De lo anterior se deduce que, el desempeño escolar es medible a través de indicadores, los cuales determinan, de alguna manera, dicho desempeño. Rodríguez y Tejedor $(1996,6)$, se refieren al rendimiento académico así:

Podemos definir dos tipos de rendimiento. Por una parte, el rendimiento en sentido estricto, medido a través de la presentación o éxito en las pruebas (calificaciones). Por otra, el rendimiento en sentido amplio, medido a través del éxito (finalización puntual), el retraso o el abandono en los estudios. También se habla de "regularidad académica", cuando el concepto de rendimiento académico se operacionaliza mediante las tasas de presentación o no a las convocatorias de exámenes.

Indudablemente para el desempeño escolar no solamente se tiene en cuenta las evaluaciones acumuladas y calificadas con un promedio de las notas obtenidas, también es necesario considerar los procesos de formación, permitiendo determinar los avances, cultivando habilidades y destrezas, evitando siempre que el conocimiento sea memorístico, formando estudiantes con mentes capaces de hacer crítica.

Según Paz $(2007,27)$ "el desempeño escolar es la resultante del complejo mundo que envuelve al estudiante: capacidades individuales, su medio sociofamiliar, su realidad escolar y por lo tanto su análisis resulta complejo y con múltiples interacciones", esto significa que el docente debe saber que el desempeño escolar depende en cierta parte del ambiente socio-familiar que lo rodea, desde su niñez ese medio social que más directamente incide en él es su familia.

Para un buen desempeño escolar se debe tener en cuenta el contexto, en el que esta inmersa la familia y las expectativas que esta tiene respecto a la educación, además de las diferentes situaciones que el estudiante enfrenta cada día, tiene presente características de la población que asiste a la escuela, para hacer cambios, buscando siempre el respeto a las diferencias, conociendo sus necesidades y capacidades dejando atrás juzgamientos.

Se vuelve a la importancia de involucrar a la familia en el proceso de formación de los sujetos para que obtengan un adecuado desempeño escolar, es importante para la relación padres-hijos la demostración constante de que el aprendizaje es una parte natural de la vida -alegre por derecho propio, es parte de la experiencia familiar, y especialmente gratificante cuando se produce a través del descubrimiento en museos, zoos y lugares históricos. (Redding, sf: 11) 
Es importante la motivación hacia el estudio, es decir, por aprender algo nuevo a través de una fuerza interna que impulsa a la persona hacia el logro de un objetivo. Aprender no solo es comprender y apropiarse de conocimientos, es estar dispuestos a reaprender constantemente.

Para el desarrollo de la presente investigación se toma al promedio de las calificaciones de la población estudiada como un indicador del desempeño escolar, es decir a mayor calificación, mayor es el desempeño escolar y de igual manera a menor calificación menor se considera el desempeño escolar.

\section{Tipo de investigación y metodología}

Esta investigación es cuantitativa porque se sirve de números y métodos estadísticos para la obtención de información; se cuantificaron los datos en cada una de las variables. Es de tipo correlacional, porque se buscó conocer la relación entre las variables: prácticas educativas familiares y desempeño escolar, es decir, se busca establecer relaciones entre dos variables. Los datos se generaron con una escala Likert y se analizaron con el software SPSS.

\section{Procedimiento}

A continuación, se especifican los pasos llevados a cabo para poder ejecutar la Investigación desde la selección de la población objeto de estudio, las respectivas solicitudes y permisos tanto de madres de familia como de la institución hasta la aplicación de la prueba, la tabulación de datos para finalmente presentar los resultados, las conclusiones y recomendaciones

\section{Población y muestra}

De una población de 377 niñas de los grados cuartos de básica primaria, jornada de la tarde, de la I.E.M. María Goretti, se tomó una muestra no probabilística, de tipo intencional, de 208 niñas, para que sus madres o acudientes respondieran el instrumento.

\section{Técnicas e instrumentos}

De las investigaciones Prácticas Alfabetizadoras Familiares Medellín (Colombia) y Sociedad, Familia y Aprendizaje La Plata, provincia de Buenos Aires (Argentina) se tomo la escala Likert, la cual fue adaptada para la presente investigación.

Se transformaron algunas preguntas y se organizaron en tres variables: Actividades de Aprendizaje, la cual comprende 8 preguntas, Prácticas Educativas Familiares del Lenguaje, con 17 preguntas y Hábitos en el Hogar, consta de 5 preguntas. Para su confiabilidad y validez se entrego el instrumento en dos ocasiones a la misma población de estudio.

Se hicieron los primeros contactos con las familias seleccionadas para participar y se les explicó la propuesta de investigación, posteriormente se entregó el instrumento a las madres para que lo respondieran durante la reunión convocada para tal fin.

Para la variable desempeño escolar se tuvieron en cuenta las calificaciones de todas las áreas del grado cuarto de las estudiantes.

Se procedió a realizar la codificación en cada una de las categorías, para después hacer una base de datos, la cual fue utilizada para la tabulación y análisis de resultados por medio del programa estadístico Statistical Package for the Social Sciences (SPSS). La sistematización de los datos se consolido a través de tablas, posteriormente se realizó una descripción, orientada a la interpretación de estas para dar paso a la discusión y por último elaborar las conclusiones y recomendaciones.

\section{Hallazgos}

Para cumplir el objetivo de esta investigación: Establecer las relaciones entre Prácticas Educativas Familiares y Desempeño Escolar en las estudiantes de los grados cuartos de básica primaria, de la Institución Educativa Municipal María Goretti se tuvieron en cuenta las respuestas 
de las madres o acudientes que recibieron el instrumento y las calificaciones de las estudiantes.

Tabla 1. Edad de las madres

\begin{tabular}{|l|l|l|}
\hline & & Edad de la madre \\
\hline $\mathbf{N}$ & Válidos & 207 \\
\hline & Perdidos & 1 \\
\hline Media & & 35,52 \\
\hline Mediana & & 34 \\
\hline Desv. Típ. & & 6,297 \\
\hline
\end{tabular}

La tabla 1, muestra que la población estudio estuvo constituida por 208 madres. La edad de las madres está entre los 26 y 56 años, con una media de 35,5 años; una mediana de 34 años, lo que representa que la mitad de ellas sobrepasa los 34 años y la otra mitad es más joven; la desviación típica es de 6,297, esto indica que las edades de las madres se desvían poco más de 6 años de la media (35.5).
Como se puede observar en la tabla 2 en las familias estudiadas, predomina el estrato socioeconómico bajo con 175 familias (84.1\%), seguido del estrato medio, con 30 familias $(14.4 \%)$ y tan solo 3 familias $(1,4 \%)$ que pertenecen a un estrato alto. Lo que significa que, de la población objeto de estudio $98.5 \%$ pertenecen a un estrato bajo y medio, lo que indica que las estudiantes tienen condiciones socioeconómicas similares.

En la tabla 3, se encuentra que 165 niñas tienen un desempeño alto y superior, es decir que 43 niñas de 208 tienen un promedio entre básico y bajo, lo cual confirma que este grupo de estudio tiene un desempeño escolar en su mayoría alto.

De lo anterior se puede afirmar que las materias con promedio más alto están en las estudiantes de estrato socioeconómico alto en las áreas: ética y valores con un promedio de notas de $(5,0)$; castellano con un promedio de notas de $(4,6)$, educación física con un promedio de notas de $(4,6)$,

Tabla 2. Estrato Socioeconómico

\begin{tabular}{|c|c|c|c|c|c|}
\hline \multicolumn{7}{|c|}{ ESTRATO SOCIO ECONÓMICO } \\
\hline & & FRECUENCIA & PORCENTAJE & PORCENTAJE VALIDO & PORCENTAJE ACUMULADO \\
\hline Validos & Bajo & 175 & 84,1 & 84,1 & 84,1 \\
\hline & Medio & 30 & 14,4 & 14,4 & 98,6 \\
\hline & Alto & 3 & 1,4 & 1,4 & 100 \\
\hline & Total & 208 & 100 & 100 & \\
\hline
\end{tabular}

Tabla 3. Desempeño Escolar

\begin{tabular}{|l|l|c|c|c|c|}
\hline \multicolumn{5}{|c|}{ Desempeño } \\
\hline & & Frecuencia & Porcentaje & Porcentaje valido & Porcentaje acumulado \\
\hline Válidos & Bajo (0-2.9) & 2 & 1 & 1 & 1 \\
\hline & Básico (3.0 - 3.7) & 41 & 19,7 & 19,7 & 20,7 \\
\hline & Alto $(3.8-4.5)$ & 149 & 71,6 & 71,6 & 92,3 \\
\hline & Superior (4.6 - 5.0) & 16 & 7,7 & 7,7 & 100 \\
\hline & Total & 208 & 100 & 100 & \\
\hline
\end{tabular}

Tabla 4. Estrato Socioeconómico y Desempeño Escolar

\begin{tabular}{|c|c|c|c|c|c|c|c|c|c|c|c|}
\hline & $\begin{array}{l}\text { Calificación } \\
\text { h educaciór } \\
\text { religiosa } \\
\end{array}$ & $\begin{array}{l}\text { Talificación } \\
\text { en etica y } \\
\text { valores }\end{array}$ & $\begin{array}{l}\text { Ealificación } \\
\text { n ciencias } \\
\text { sociales }\end{array}$ & $\begin{array}{l}\text { Calificación } \\
\text { naturales } \\
\text { naiencias }\end{array}$ & $\begin{array}{c}\text { Calificación } \\
\text { en } \\
\text { hatemáticas }\end{array}$ & $\begin{array}{l}\text { Calificacione } \\
\text { en ingles }\end{array}$ & $\begin{array}{l}\text { Calificación } \\
\text { n castelland }\end{array}$ & $\begin{array}{l}\text { Calificación } \\
\text { n tecnologis }\end{array}$ & $\begin{array}{l}\text { Calificación } \\
\text { n educación } \\
\text { fisica }\end{array}$ & \begin{tabular}{|c|} 
Calificación \\
n educación \\
artistica
\end{tabular} & $\begin{array}{l}\text { Promedio de } \\
\text { las } \\
\text { alificacione }\end{array}$ \\
\hline & Media & Media & Media & Media & Media & Media & Media & Media & Media & Media & Media \\
\hline Estrato socioecor Bajo (1y & 4,3 & 4,2 & 3,8 & 3,7 & 3,6 & 3,7 & 4,0 & 4,0 & 4,6 & 4,1 & $\overline{4,0}$ \\
\hline Medio (s) & 4,3 & 4,3 & 3,9 & 3,8 & 3,6 & 3,7 & 4,1 & 4,0 & 4,5 & 4,3 & 4,0 \\
\hline Alto $(4 y)$ & 4,4 & 5,0 & 4,3 & 4,3 & 4,4 & 4,3 & 4,6 & 4,5 & 4,6 & 4,5 & 4,5 \\
\hline
\end{tabular}


en estudiantes de estratos alto y bajo. Por otra parte, el promedio de notas más bajo esta en la materia de matemáticas y lo tienen los estratos socioeconómicos bajo y medio con $(3,6)$. Es necesario aclarar que el número de estudiantes de estrato social alto son tres.

Las familias que pertenecen al estrato medio son quienes más utilizan las prácticas educativas familiares. Sin embargo estas se presentan en los demás estratos, pero con una media más baja.

A continuación se empieza a cruzar las categorías. En las tablas se señala en el caso de correlación con uno o dos asteriscos. Para entender mejor el análisis, es importante recordar que se presenta correlación cuando es de 0,05 o 0,01 bilateral.

Dado que sig. >0.05 en la comparación del rendimiento académico con las variables asociadas con las practicas educativas familiares, se concluye que no existe correlación estadísticamente significativa. Es decir, las prácticas educativas familiares no se correlacionan con el desempeño escolar. No obstante, Se aprecia correlación entre las diferentes prácticas educativas familiares Sig. $<0.05$.

Esto significa que las prácticas educativas familiares tienen correlación una en la otra, es decir que todas de algún modo están presentes sin llegar a impactar el desempeño académico.

Al revisar la literatura se hallaron trabajos que analizan diferentes prácticas de la familia de los estudiantes y la relacionan con el desempeño escolar de los mismos.

Como objetivo general de esta investigación, se buscó establecer relaciones entre prácticas educativas familiares y desempeño escolar en básica primaria el primer aspecto que se consideró en las prácticas educativas familiares fueron las actividades de aprendizaje, se encontró que la familia tiene prácticas educativas familiares como las actividades de aprendizaje, sin embargo, como se explica en la estadística de esta investigación, estas actividades no inciden en el desempeño escolar de las estudiantes del grupo objeto de estudio.

No cabe duda que, en todos estos procesos un factor importante es la familia como actor principal de la formación de los hijos, en ella además de la formación se definen aspectos que inciden en su vida adulta, de igual manera en la autoestima y en la autonomía, asuntos q u e también son fundamentales en la formación de la personalidad de los sujetos y en su desempeño escolar. Cada familia desarrolla actividades teniendo en cuenta sus características culturales, sociales y personales, como parte de su formación y esto incluye actividades de aprendizaje, donde se comienza de igual forma, a hacer una representación del mundo, tal como lo afirma Aguilar (2005).

El segundo aspecto considerado fue las prácticas educativas familiares del lenguaje, el ser humano en todo momento necesita el lenguaje para comunicarse. La oralidad como primera forma de comunicación, da origen a la escritura. El lenguaje escrito por medio de la lectura y la escritura, acompaña a las personas durante toda su vida, sin olvidar que existen otras formas de comunicación, sin embargo en el presente estudio se hace énfasis tanto

Tabla 5. Estrato socioeconómico y Prácticas Educativas Familiares

\begin{tabular}{|c|c|c|c|c|c|c|c|c|c|}
\hline & \multicolumn{3}{|c|}{ Actividades de aprendizaje } & \multicolumn{3}{|c|}{$\begin{array}{l}\text { Prácticas educativas } \\
\text { familiares del lenguaje }\end{array}$} & \multicolumn{3}{|c|}{ Hábitos en el hogar } \\
\hline & Media & Mediana & $\begin{array}{l}\text { Desv. } \\
\text { Típica }\end{array}$ & Media & Mediana & $\begin{array}{l}\text { Desv. } \\
\text { típica }\end{array}$ & Media & Mediana & $\begin{array}{l}\text { Desv. } \\
\text { típica }\end{array}$ \\
\hline \multirow{3}{*}{$\begin{array}{l}\text { Estrato. Bajo (1 y 2) } \\
\text { Medio (3) } \\
\text { Alto (4 y } 5)\end{array}$} & 56,01 & 57,50 & 20,62 & 69,54 & 71,76 & 17,94 & 56,75 & 56,00 & 23,18 \\
\hline & 57,33 & 57,50 & 19,02 & 72,16 & 71,18 & 15,04 & 58,93 & 64,00 & 25,12 \\
\hline & 43,33 & 40,00 & 8,04 & 65,48 & 56,47 & 17,70 & 56,00 & 64,00 & 28,84 \\
\hline
\end{tabular}


Tabla 6. Correlación de Pearson: Prácticas Educativas Familiares y Desempeño Escolar

\begin{tabular}{|c|c|c|c|c|c|c|}
\hline \multicolumn{7}{|c|}{ CORRELACIONES } \\
\hline & & & $\begin{array}{l}\text { Promedio de } \\
\text { calificaciones }\end{array}$ & $\begin{array}{l}\text { Actividades } \\
\text { de } \\
\text { aprendizaje }\end{array}$ & $\begin{array}{c}\text { Prácticas } \\
\text { educativas } \\
\text { familiares del } \\
\text { lenguaje } \\
\end{array}$ & $\begin{array}{c}\text { Hábitos en el } \\
\text { hogar }\end{array}$ \\
\hline \multirow{4}{*}{ 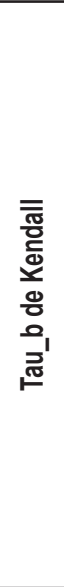 } & $\begin{array}{l}\text { Promedio de las } \\
\text { calificaciones }\end{array}$ & $\begin{array}{l}\text { Correlación de Pearson } \\
\text { Sig (bilateral) } \\
\text { N }\end{array}$ & $\begin{array}{r}1,000 \\
208 \\
\end{array}$ & $\begin{array}{r}, 028 \\
\\
, 574 \\
208 \\
\end{array}$ & $\begin{array}{l}, 031 \\
\\
, 522 \\
208 \\
\end{array}$ & $\begin{array}{r}, 022 \\
, 661 \\
208 \\
\end{array}$ \\
\hline & $\begin{array}{l}\text { Actividades de } \\
\text { aprendizaje }\end{array}$ & $\begin{array}{l}\text { Correlación de Pearson } \\
\text { Sig (bilateral) } \\
N\end{array}$ & $\begin{array}{l}, 028 \\
\\
, 574 \\
208 \\
\end{array}$ & 1,000 & $\begin{array}{r}, 462^{* *} \\
, 000 \\
208 \\
\end{array}$ & $\begin{array}{r}, 315^{* \star} \\
, 000 \\
208\end{array}$ \\
\hline & $\begin{array}{l}\text { Prácticas } \\
\text { educativas } \\
\text { familiares del } \\
\text { lenguaje }\end{array}$ & $\begin{array}{l}\text { Correlación de Pearson } \\
\text { Sig (bilateral) } \\
\text { N }\end{array}$ & $\begin{array}{r}, 031 \\
\\
.522 \\
208 \\
\end{array}$ & $\begin{array}{r}, 462^{* \star} \\
, 000 \\
208 \\
\end{array}$ & 1,000 & $\begin{array}{r}, 435^{\star \star} \\
, 000 \\
208 \\
\end{array}$ \\
\hline & $\begin{array}{l}\text { Hábitos en el } \\
\text { hogar }\end{array}$ & $\begin{array}{l}\text { Correlación de Pearson } \\
\text { Sig (bilateral) } \\
\text { N }\end{array}$ & $\begin{array}{l}, 022 \\
\\
, 661 \\
208 \\
\end{array}$ & $\begin{array}{r}.315^{\star \star} \\
\\
, 000 \\
208 \\
\end{array}$ & $\begin{array}{r}., 435^{\star *} \\
\\
, 000 \\
208 \\
\end{array}$ & 1,000 \\
\hline \multirow{4}{*}{ 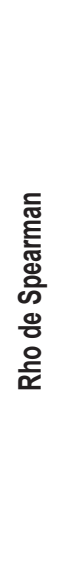 } & $\begin{array}{l}\text { Promedio de las } \\
\text { calificaciones }\end{array}$ & $\begin{array}{l}\text { Correlación de Pearson } \\
\text { Sig (bilateral) } \\
\text { N }\end{array}$ & $\begin{array}{r}1,000 \\
208 \\
\end{array}$ & $\begin{array}{r}, 040 \\
\\
, 565 \\
208 \\
\end{array}$ & $\begin{array}{l}, 044 \\
\\
, 527 \\
208 \\
\end{array}$ & $\begin{array}{r}, 032 \\
\\
, 645 \\
208 \\
\end{array}$ \\
\hline & $\begin{array}{l}\text { Actividades de } \\
\text { aprendizaje }\end{array}$ & $\begin{array}{l}\text { Correlación de Pearson } \\
\text { Sig (bilateral) } \\
\text { N }\end{array}$ & $\begin{array}{l}, 040 \\
\\
, 585 \\
208 \\
\end{array}$ & 1,000 & $\begin{array}{r}.625^{* *} \\
, 000 \\
208 \\
\end{array}$ & $\begin{array}{r}, 440^{* *} \\
, 000 \\
208 \\
\end{array}$ \\
\hline & $\begin{array}{l}\text { Prácticas } \\
\text { educativas } \\
\text { familiares del } \\
\text { lenguaje }\end{array}$ & $\begin{array}{l}\text { Correlación de Pearson } \\
\text { Sig (bilateral) } \\
N\end{array}$ & $\begin{array}{l}, 044 \\
\\
.527 \\
208 \\
\end{array}$ & $\begin{array}{r}, 625^{* *} \\
, 000 \\
208 \\
\end{array}$ & 1,000 & $\begin{array}{r}, 604^{* *} \\
, 000 \\
208 \\
\end{array}$ \\
\hline & $\begin{array}{l}\text { Hábitos en el } \\
\text { hogar }\end{array}$ & $\begin{array}{l}\text { Correlación de Pearson } \\
\text { Sig (bilateral) } \\
\text { N }\end{array}$ & $\begin{array}{l}, 032 \\
\\
645 \\
208 \\
\end{array}$ & $\begin{array}{r}, 440 * * \\
, 000 \\
208\end{array}$ & $\begin{array}{r}, 604^{* *} \\
, 000 \\
208 \\
\end{array}$ & 1,000 \\
\hline
\end{tabular}

en el lenguaje escrito, como en el lenguaje oral, pero no se encuentra correlación de las prácticas educativas familiares del lenguaje con el desempeño escolar.

El niño siempre está rodeado de lenguaje, es fundamental que la familia se interese por la lectura y la escritura, porque es el lugar donde se comienzan a desarrollarlas, es la familia la encargada de guiar al niño al mundo del lenguaje, y las prácticas educativas familiares, que aunque muchas veces no se hacen de forma premeditada, son la base fundamental para el desarrollo del proceso de aprendizaje del lenguaje oral y escrito.

La literatura muestra lo importante que es practicar el lenguaje escrito en el hogar para mejorar el aprendizaje según Gómez (2001, 93) "Las prácticas de lectura y escritura de los padres también parecen estar relacionadas con el rendimiento académico de los hijos." Este autor ya 
relaciona el desempeño escolar con las prácticas de lectura y escritura. También para Carrasco $(2007,178)$ "Los estudios han relacionado positivamente ciertas prácticas familiares con el aprendizaje del niño, algunas se refieren a la relación padre-hijo (expresiones de afecto, conversar diariamente, hacer comentarios en familia sobre libros, revistas, visitas a museos)". Este autor, además de las prácticas de lectura y escritura tiene en cuenta otros factores como la relación que existe entre los padres y los hijos al respecto de lo académico.

El tercer aspecto es hábitos en el hogar. "Los hábitos y actitudes observadas en los padres influyen en la adquisición de hábitos y en el desarrollo de actitudes por los hijos en edades tempranas" (Gil, $2009,316)$. Es decir que los hábitos y las rutinas son habilidades que se adquieren desde los hogares, para la realización de determinados actos, lo que significa que son las repeticiones de diferentes actividades que conllevan a resultados positivos, en el caso de estudiantes puede ser, un alto desempeño escolar. "Los resultados confirman mayores niveles de competencia en el alumnado cuyos padres presentan mejores actitudes hacia la lectura y declaran dedicar mayor número de horas semanales a esta actividad" (301).

Esta investigación presenta como hipótesis de trabajo: A mayor utilización de prácticas educativas familiares, mejor desempeño escolar en los estudiantes, sin embargo los resultados que se obtuvieron, muestran que las prácticas educativas familiares están relacionadas entre ellas, pero no se relacionan con el desempeño escolar, posiblemente el desempeño escolar depende de otras variables que no se tuvieron en cuenta en este proceso investigativo, por tal razón, se probó la hipótesis nula: las prácticas educativas familiares no influyen en el desempeño escolar.

La segunda variable es el desempeño escolar, se refiere a la evaluación integral de los conocimientos adquiridos en el contexto escolar, el cual va a ser alto cuando las calificaciones de los exámenes o actividades escolares son positivas, se puede decir que es una forma de medir lo que ha aprendido el estudiante a lo largo de su proceso de formación.

A pesar de existir investigaciones en las que se encuentra una relación positiva entre desempeño escolar y las prácticas educativas familiares, esta investigación arroja resultados que contradicen en parte estos postulados, puesto que si bien es cierto que las practicas educativas familiares se correlacionan entre sí, los resultados en este grupo de investigación, las prácticas educativas familiares no tienen correlación con el desempeño escolar; lo que llama la atención es ¿por qué en este grupo las prácticas educativas familiares no tienen relación con el desempeño escolar, aunque se si entre ellas? ¿Qué es entonces, lo que determina el desempeño escolar?, ¿puede ser que los métodos de evaluación, no abarcan realmente todos los aspectos que comprenden un proceso de formación educativa?

De acuerdo a los resultados encontrados, se puede afirmar que las prácticas que se estudiaron en esta investigación, no tienen correlación con el desempeño escolar; sin embargo, se encontró otros factores si se correlacionan positivamente en el desempeño escolar como la edad de las madres. Entre más jóvenes brindan mayor apoyo a sus hijos. En relación con el estrato socioeconómico, a mayor estrato socioeconómico mayor desempeño escolar, no obstante es necesario recordar que las familias de estrato alto son tres.

En cuanto a los objetivos específicos de la investigación. Se encuentra que 165 niñas que equivale al $79.3 \%$, tienen un desempeño alto y superior; 43 niñas, $20,7 \%$ tienen un promedio entre básico y bajo, lo cual confirma que este grupo de estudio tiene un desempeño escolar en su mayoría alto. 


\section{Conclusiones}

A partir de la información recolectada se puede concluir lo siguiente:

Para determinar la correlación entre las prácticas educativas familiares y desempeño escolar se debe tomar en cuenta: estados momentáneos o permanentes del sujeto, el contexto en el cual se desarrolla, estados físicos y sociales, además de las expectativas de las estudiantes.

La investigación se realizó con un grupo no representativo, razón por la cual no se considera significativo para obtener información que permita ser generalizada para la Institución o la ciudad de Pasto, pero si permite encontrar una aproximación al comportamiento de las variables escogidas, para hacer una generalización se tendría que trabajar con una muestra estadística, de toda la Institución, o también se podría trabajar con una muestra probabilística o aleatoria de varias instituciones de la ciudad de Pasto. Sin embargo, el objetivo de la investigación estaba dirigido a determinar la correlación entre las prácticas educativas familiares y el desempeño escolar de un grupo no representativo de básica primaria de la I.E.M. María Goretti, lo que se cumplió a cabalidad.

Las prácticas educativas familiares son diversas y es posible que las que se tuvieron en cuenta en la presente investigación, no sean suficientes para apoyar el desempeño escolar, porque así mismo son múltiples los factores que intervienen en este como, nutrición de las estudiantes, metodología utilizadas para la enseñanza, los factores asociados a la inteligencia de las estudiantes y a las capacidades de aprendizaje, los aspectos emocionales y los intereses tanto de los padres de familia como de las niñas en cuanto a su futuro académico, el estado emocional de las estudiantes en el transcurso de año escolar, los factores sociales de las niñas en el momento de cursar el año estudiado, las expectativas o proyectos de vida que las estudiantes tengan, las estrategias de manejo del estrés o las estrategias de solución de problemas que tenga cada estudiante, entre otras.

El instrumento de investigación debió ser aplicado a padres y madres de familia, al ser entregado únicamente a las madres, se excluyó a los padres, quienes posiblemente sean generadores de prácticas educativas familiares que las madres no realizan.

\section{Recomendaciones}

Teniendo en cuenta que la población que participa en esta investigación en su mayoría son madres jóvenes, se puede plantear un programa de educación continuada y de actualización de tal modo que se posibilite mayor participación de las madres en la educación de las hijas, que permita al mismo tiempo, contribuir al mejoramiento de las condiciones de vida de las familias de las estudiantes de la I.E.M María Goretti.

El desempeño escolar de las estudiantes de la I.E.M. María Goretti que participaron en la investigación, es alto y superior, se pueden plantear estrategias para que este promedio se mantenga en este rango.

Después de comprobar, por medio del presente trabajo investigativo, que las prácticas educativas familiares presentes en los hogares de las estudiantes de grado cuarto de la Institución Educativa Municipal María Goretti, no tienen correlación con el desempeño escolar, se debe plantear otras investigaciones que permita identificar cuáles son los factores que realmente se correlacionan con el desempeño escolar, y determinar cuál es la función de las prácticas educativas familiares.

Finalmente, se recomienda identificar otros aspectos diferentes a las prácticas educativas que se correlacionen con el desempeño escolar en forma directa, para poder trabajar sobre estas, en búsqueda de encontrar un mejoramiento en la vida educativa de las estudiantes. 


\section{Bibliografía}

Aguilar Ramos, Maria Carmen (2005). Las prácticas educativas en el ámbito familiar. Universidad de Málaga. p. 1-10

Alfonso, Carmen, Amat, Roser, (2003) La participación de los padres y madres en la escuela. Caracas: Editorial. Laboratorio educativo

Alguacil de Nicolás; Pañellas, Montserrat, (2009) Implicación de las Familias en los Institutos de Enseñanza Secundaria. En: http://www.javeriana.edu.co/magis/numerotres/PDFs/5_monserrat_alguacil.pdf (Recuperado en octubre 8 de 2011 )

Alonso García, Julia; Román Sánchez, José María. (2005). Prácticas educativas familiares y autoestima. Psicothema. Vol17 (1). p. 76-82.

Araiza, Vanessa, Silva, Arturo, Coffin, Norma \& Jiménez, Lourdes. 2009. Hábitos de estudio, ambiente familiar y su relación con el consumo de drogas en estudiantes. México

Armesto, Celia. (2000) Educación Infantil Familiar: Una utopía realizable. Madrid: Editorial Cartas Española Editores.

Beltrán, Jesús; Bueno, José. (1995) Psicología de la Educación. Barcelona: Editorial Bolxareu Universitaria Marcombo

Botero Gómez, Patricia; Salazar Henao, Myriam; Torres, María Leticia. (2009). Narrativas y prácticas de crianza: hacia la construcción de relaciones vinculantes, lo público y la democracia frente a la violencia intrafamiliar en ocho OIF de Caldas. Centro de Estudios Avanzados en Niñez y Juventud, alianza CINDE Universidad de Manizales en convenio con ICBF, Regional Caldas. Colombia.

Carrasco, José. (2007). Cómo personalizar la educación, una solución de futuro. BarceIona: Editorial Narcea, S.A. De Ediciones.

Cerillo, Pedro; Larrañaga, Elisa; Yubero, Santiago. (2002) Libros, lectores y mediadores: la formación de los hábitos lectores como proceso de aprendizaje. Cuenca: Ediciones de la universidad de castilla. La mancha.

Espitia, Rosa Elena; Montes, Marivel. (2009) Influencia de la familia en el proceso educativo de los menores del barrio costa azul de Sincelejo (Colombia) En: http:// ciruelo.uninorte.edu.co/pdf/invest_desarroIlo/17-1/04\%20INFLUENCIA\%20DE\%20 LA\%20FAMILIA.pdf (Recuperado en octubre 20 de 2011)

Estévez, Estefanía; Jiménez, Terebel; Musitu, Gonzalo. (2007). Relaciones entre padres e hijos adolescente. Cuenca: Editorial Nau Llibres.

Gary, Kielhofner. (2004) Terapia ocupacional, modelo de ocupación humana. Tercera edición. Buenos Aires: Editorial Médica Panamericana S.A.

Gil, del Carmen. (2008). Una propuesta de Integración Social desde la escuela. Sevilla: Editorial Mad S.L.

Gil Flores, Javier. (2009). Hábitos y actitudes de las familias hacia la lectura y competencias básicas del alumnado. Universidad de Sevilla. Facultad de Ciencias de la Educación. Departamento de Métodos de Investigación y Diagnóstico en Educación (MIDE). Sevilla.

Gómez, Carmen. (2001) Identidades de género y feminización del éxito académico, Ministerio de Educación Cultura y Deporte, Secretaría general de Educación y Formación profesional, Centro de investigación y documentación Educativa (CIDE). Colección Investigación número 151. Sevilla: Edita Secretaria general técnica.

Gonzáles, Daniel. (2005).El Desempeño Académico Universitario, variables psicológicas. Secretaría de Educación Pública, Universidad de Sonora. Sonora.

González, Julio; Núñez, José; Álvarez, Luis; Roces, Cristina; González, Soledad; González, Paloma; Muñiz, Roberto; o Valle, Antonio; Cabanach, Ramón; Rodríguez, Susana; Bernardo, Ana. (2003). Adaptabilidad y cohesión familiar, implicación parental en conductas autor regulatorias, auto concepto del estudiante y rendimiento académico. En: http://www.psicothema.es/ pdf/1090.pdf. (Recuperado en noviembre 13 de 2011)

Henao López, Gloria Cecilia; García Vesga, María Cristina. (2009). Revista latinoamericana de ciencias sociales, niñez y juventud. Universidad de Manizales. Vol. 7 (2). p. 785-802. 
Henao López, Gloria Cecilia; Ramírez Palacio, Carlota; Ramírez, Luz Ángela. (2007). Artículo de investigación, Las prácticas educativas familiares como facilitadoras del proceso de desarrollo en el niño y niña. Grupo de Estudios Clínicos y Sociales en Psicología Universidad de San Buenaventura, pg. 233- 240

Hincapié Tamayo, Sandra. (2011) Prácticas alfabetizadoras familiares. En: http://bibliotecadigital.usb.edu.co (Recuperado en noviembre 27 de 2011)

Kelly, William. (1982) Psicología de la educación: psicopedagogía fundamental y didáctica. Séptima edición. Barcelona: Editorial Morata.

Marín, María Gabriela. (2004). Alumnos con necesidades educativas especiales. San José de Costa Rica: Editorial Universal Estatal a distancia.

Mayor, Cristina; Rodríguez, José. 1996. Hábitos de estudio y trabajo intelectual en estudiantes del magisterio. Barcelona.

Ortega, Justo. (2006). Bajo rendimiento escolar, Bases emocionales de su origen y vías afectivas para su tratamiento. Barcelona: Primera edición. Incipit editores.

Panza, Margarita. (1991). Hábitos y técnicas de estudio aprender es cosa fácil. México.

Paz, Sofía. (2007). Problemas en el desempeño escolar y su relación con el funcionalismo familiar en alumnos de EGB 1. Revista de la facultad de medicina. Vol. 8 - no 1. p. 27-32.

Peralbo Uzquiano, Manuel; Fernández Amado, María Luz. (2003) Revista Galego-Portuguesa de Psicología educación estructura familiar y rendimiento escolar en Educación secundaria obligatoria $n^{\circ} 7$ (vol. 8) ano $7^{\circ}$ 2003 issn: 1138-1663. En: http://ruc.udc.es/ dspace/bitstream/2183/6933/1/RGP_9-23. pdf (Recuperado en diciembre 9 de 2011 )
Querejeta, Maira. (2010) Orientación y Sociedad, Avances de investigación, Sociedad, familia y aprendizaje. El papel de los contextos hogareños. En: http://www.scielo.org. ar/pdf/orisoc/v10/v10a05.pdf (Recuperado en diciembre 12 de 2011)

Redding, Sam. (S, f.). Familias y centros escolares Academia Internacional de Educación Oficina Internacional de Educación. En: http://www.ibe.unesco.org/fileadmin/ user_upload/archive/publications/EducationaIPracticesSeriesPdf/prac02s.pdf (Recuperado en diciembre 15 de 2011)

Redding, Sam. (S, f.). Familias y centros escolares Academia Internacional de Educación Oficina Internacional de Educación. Serie de prácticas educativas No. 3 Prácticas eficaces University of Illinois en Chicago.

Rodríguez, José; Tejedor, Francisco. (1996). Documentos didácticos Evaluación educativa I. Evaluación de los aprendizajes de los alumnos.

Rodríguez, Socorro. (2005) Salud mental del niño de 0 a 12 años, Necesidades básicas del niño. San José de Costa Rica: Editorial Universal Estatal a distancia.

Valderrama, Milady; Behn Verónica; Pérez, María; Díaz, Alejandro; Henríquez, Patricia; Torruella, Matilde. (2005). Factores de Riesgo Biopsicosocial que Influyen en el Fracaso Escolar en Alumnos Vulnerables de Escuelas Municipalizadas de la Comuna de San Pedro de la Paz. En http://www.scielo. cl/pdf/cienf/v13n2/art06.pdf (Recuperado en noviembre 15 de 2011)

Vila, Ignasi. (1998) Escuela y comunidad, Instituto de Ciencias de Educación; Universidad de Barcelona: Editorial Horsori http:// definicion.de/rendimiento-academico/ (Recuperado en septiembre 11 de 2011). 